\title{
Online-only primary care services - learning from CQC inspection
}

\author{
Authors: Ayse Sema ${ }^{A}$ and James Clemance ${ }^{B}$
}

\begin{abstract}
Aims
Remote consultation through online-only channels, for example video or text (web forms), is a rapidly growing healthcare sector. The Care Quality Commission (CQC) has seen year-on-year growth in applications for such services in England since 2010 ( 40 such services are registered). While these providers are mainly in the independent sector, there are collaborations with the NHS to deliver remote consultation, and de novo implementation of online consultation modalities within general practitioner practices and secondary care settings.
\end{abstract}

\section{Methods}

CQC have completed a first-round inspection programme of the online primary care sector - as of 13 December 2017 five were compliant on first inspection, enforcement action was taken in 17 , requirement notices alone in 12 - in a sector new to regulation.

\section{Results}

Of 10 services with re-inspection reports, seven demonstrated improvement.

\section{Conclusions}

This work highlighted themes important for safe care and treatment - which are important for organisations adopting and developing remote consultation services in the NHS or independent sector.

> As service users access care from a variety of NHS and independent sector providers, effective and timely communication between providers is essential for quality care.

$>$ Where services are delivered remotely to new users, effective identification processes underpin safe care and treatment.

$>$ As geographic footprints of services extend beyond regional and national borders, safeguarding procedures must evolve to remain effective.

Authors: ${ }^{A}$ Strategy \& Intelligence - Engagement, Care Quality Commission; ${ }^{\mathrm{B} C Q C}$, London, UK
We will be publishing our end of programme report which looks in detail at our findings from this round of inspection in the coming months.

Drawing on the findings of this report, we would use the session to discuss the opportunities and challenges of remote service delivery, examples of notable practice, and learning for services and clinicians who might be considering implementation. 